\title{
Ansiedade, depressão e desesperança em pacientes com doença pulmonar obstrutiva crônica
}

\section{Anxiety, depression and hopelessness in patients with Chronic Obstructive Pulmonary Disease (COPD)}

\author{
Rossane Frizzo de Godoy*
}

Universidade de Caxias do Sul - UCS, Caxias do Sul, Rio Grande do Sul, Brasil

\begin{abstract}
RESUMO
A Doença Pulmonar Obstrutiva Crônica (DPOC) é altamente incapacitante. O reconhecimento de fatores emocionais comuns a esses pacientes pode fornecer subsídios importantes para os profissionais que atuam na área da saúde. Objetivos: avaliar o impacto do programa de reabilitação pulmonar (PRP) sobre os níveis de ansiedade, depressão e desesperança de pacientes com DPOC, por meio da logoterapia. Métodos: 17 pacientes foram avaliados no início e ao final do PRP com três instrumentos de medida: Inventário Beck de Ansiedade, Inventário Beck de Depressão e Escala de Desesperança de Beck. Resultados: Participaram deste estudo 17 pacientes (52,9\% homens), idade média de $64,5 \pm 8,1$ anos. Ansiedade e depressão foram constatadas em 29,4\% da amostra; desesperança em 41,1\%. Na comparação entre pré e pós testes, os resultados foram os seguintes: ansiedade $(p=0,002)$; depressão $(p=0,001)$; desesperança $(p=0,03)$. Conclusões: a realização do PRP mostrou-se efetiva para reduzir os níveis de ansiedade, depressão e desesperança dos pacientes.
\end{abstract}

Palavras-chave: Ansiedade, Depressão, Desesperança, DPOC.

\begin{abstract}
Chronic Obstructive Pulmonary Disease is a highly disabling disease. The recognition of the emotional factors common to these patients may provide important information for professionals working with such subjects. Objectives: to assess the impact of pulmonary rehabilitation program (PRP) on the levels of anxiety, depression and hopelessness in patients with COPD, through logotherapy. Methods: 17 COPD patients were assessed at baseline and at the end of PRP based on three scales: Beck Anxiety Inventory, Beck Depression Inventory and Beck Hopelessness Scale. Results: The study included 17 patients $(52.9 \%$ men) with mean age of $64.5 \pm 8.1$ years. Anxiety and depression were present in $29.4 \%$ of the sample, $41.1 \%$ in despair. In the comparison between the analyzed variables during the pre and post tests, the results were as follows: anxiety $(p=0.002)$, depression $(p=0.001)$, hopelessness $(p=0.03)$. Conclusions: The achievement of a pulmonary rehabilitation program has proven effective to reduce levels of anxiety, depression and hopelessness in patients with COPD.
\end{abstract}

Keywords: Anxiety, Depression, Hopelessness, COPD. 


\section{I ntrodução}

A Doença Pulmonar Obstrutiva Crônica (DPOC) é considerada a maior causa de morbidade e mortalidade em adultos americanos (CELLI, 2010). Trata-se uma doença altamente incapacitante, seu impacto é percebido em vários aspectos da vida dos pacientes (RIES; BAULDOFF; CARLIN; CASABURI; EMERY; MAHLER \& COLS, 2007). Dispnéia (falta de ar) consiste no principal sintoma e, refere-se à sensação experimentada pelo paciente, quando o ato de respirar passa à esfera da consciência como um esforço desagradável. É uma experiência percebida de forma complexa e altamente subjetiva. Fatores psicológicos, afetivos, fisiológicos e ambientais podem interferir nesta percepção do paciente (YORKE; MOOSAVI; SHULDHAM; JONES, 2010).

Sintomas de ansiedade e depressão são bastante comuns nos pacientes com DPOC e, geralmente aparecem como decorrência da doença. Prevalências para ansiedade e depressão apresentam variações entre 12 a $96 \%$ e $27 \%$ a $79 \%$ respectivamente (GODOY; TEIXEIRA; BECKER; MICHELLI; GODOY, 2009)

No que concerne a ansiedade percebe-se que há uma relação direta com a exigência física. Os pacientes temem que qualquer atividade física possa precipitar um episódio de falta de ar e a ansiedade aparece quando precisam se empenhar em alguma atividade. Como conseqüência, tornam-se cada vez mais inativos, agravando ainda mais a dispnéia (GODOY \& GODOY, 2003).

A depressão é considerada uma resposta psicológica na medida em que o paciente precisa se adaptar a essa nova condição. As dificuldades são visíveis em todas as áreas. Muitos pacientes aposentam-se por invalidez; outros precisam mudar de trabalho. Atividades sociais e recreativas tornam-se diminuídas, pois o paciente passa a sentir-se constrangido e estigmatizado devido à sintomatologia tornando-se recluso em sua própria casa. Mudanças nas relações afetivas, familiares e sexuais, também são referenciadas (GODOY, 2003). Um estudo com pacientes com DPOC, corroborou a relação entre a deficiência sentida pelo paciente no desenvolvimento das atividades de vida diária e o aumento nos sintomas de depressão (KATZ; JULIAN; OMACHI; GREGORICH; EISNER; YELIN ; BLANC, 2010).

Pesquisas têm mostrado uma associação entre DPOC e pobre imagem corporal, baixo autoconceito, aumento do sentimento de solidão e insatisfação com o apoio social recebido ((RIES; BAULDOFF; CARLIN; CASABURI; EMERY; MAHLER \& COLS, 2007).

Como decorrência de todas essas limitações, os pacientes apresentam sentimentos de tristeza, culpa por não poderem manter suas vidas como antes, medo da morte, sentimentos de desamparo. Um estudo observou que o desamparo foi fator preditivo no 
desenvolvimento de exacerbações em DPOC (OMACHI; KATZ; YELIN ; IRIBARREN ; KNIGHT; BLANC ; EISNER, 2010).

Com o aumento das dificuldades, alguns pacientes começam a delegar tarefas a terceiros. Aos poucos isso tudo vai contribuindo, ainda mais, para a perda da autoconfiança e conseqüente aumento da situação de dependência. Essa mudança impactante de rotina de vida do paciente agrava-se com a perda de referência de valores antes reconhecidos, podendo ocasionar a perda do sentido da vida e transformar-se num vazio existencial (FRANKL, 1985). Uma investigação realizada na China com pacientes clínicos demonstrou um aumento nas taxas de suicídio em pacientes com DPOC, constatando que das populações clínicas estudadas estes pacientes foram os mais autodestrutivos e que apresentaram maior índice de fatalidade em suas tentativas (HUNG ; LIU ; LIAO; CHANG; YANG; YEH, 2000).

O presente estudo buscou avaliar o impacto do programa de reabilitação pulmonar (PRP), terapêutica padrão recomendada pela comunidade científica para atendimento de pacientes com DPOC (RIES; BAULDOFF; CARLIN; CASABURI; EMERY; MAHLER \& COLS, 2007), sobre os níveis de ansiedade, depressão e desesperança, enfatizando as contribuições que a Logoterapia pode oferecer no atendimento psicológico desses pacientes.

\section{Método}

O estudo foi realizado no Instituto de Medicina do Esporte e Ciências Aplicadas ao Movimento Humano da Universidade de Caxias do Sul (IME-UCS), centro de referência para reabilitação pulmonar da região nordeste do Rio Grande do Sul.

A amostra foi composta por 17 pacientes com DPOC avaliados no início (pré) e ao final (pós) de PRP ocorridos entre os meses de julho de 2009 a junho de 2010. Os pacientes foram estadiados em graves e muito graves de acordo com os critérios da Global Initiative for Chronic Obstructive Disease - GOLD (RABE; HURD; ANZUETO; BARNES; BUIST; CALVERLEY \& COLS, 2007).

Os instrumentos de medida descritos a seguir foram aplicados pelo mesmo psicólogo de maneira individual no início e ao final da realização do PRP:

1 - Inventário Beck de Ansiedade (BAI): Esse inventário foi proposto por Beck para medir os sintomas comuns de ansiedade.O BAl consta de uma lista de 21 sintomas com quatro alternativas cada um, em ordem crescente do nível de ansiedade. $O$ paciente opta por aquela que lhe parecer mais apropriada. O inventário é auto-aplicável. A soma dos escores 
identifica o nível de ansiedade. Esse instrumento foi validado no Brasil, com a seguinte classificação: zero a 10 - Mínimo; 11 a 19 - Leve; 20 a 30 - Moderado e 31 a 63 - Grave (CUNHA, 2001).

2 - Inventário de Beck para Depressão (BDI): Esse inventário foi proposto por Beck por constituir-se numa forma objetiva para se medir as manifestações comportamentais da depressão. O inventário utilizado compreende 21 categorias de sintomas e atividades, com 4 alternativas cada um, em ordem crescente do nível de depressão. O paciente escolhe a que the parecer mais apropriada. O BDI é auto-aplicável. A soma dos escores identifica o nível de depressão. Esse instrumento foi validado no Brasil com a seguinte classificação: zero a 11- mínimo; 12 a 19leve; 20 a 35- moderado e 36 a 63- Grave. (CUNHA, 2001).

3 - Escala de Desesperança de Beck (BHS). É uma escala dicotômica proposta por Beck que engloba 20 itens consistindo em afirmações que envolvem cognições sobre desesperança. $O$ paciente deve concordar ou discordar da afirmação, permitindose avaliar a extensão das expectativas negativas que o paciente tem com respeito ao futuro imediato e remoto. A soma dos escores identifica o nível de desesperança. Os escores variam de 0 a 20. Esse instrumento foi validado no Brasil com a seguinte classificação: zero a 4- mínimo; 5 a 8-leve; 9 a 13moderado e 14 a 20- grave. (CUNHA, 2001).

Protocolo de atividades do PRP

Os participantes inseridos neste estudo realizaram um PRP de duração de 12 semanas composto pelas seguintes atividades: a) três sessões semanais de exercícios físicos sob responsabilidade do profissional de educação física, com duração de uma hora; b) três encontros semanais com o fisioterapeuta para trabalhar a reeducação respiratória c) uma sessão de educação mensal realizada pelo pneumologista para discutir tópicos importantes sobre DPOC e, d) uma sessão de psicoterapia grupal semanal, realizada pela psicóloga . Nas sessões de grupo foram trabalhadas as necessidades psicológicas dos pacientes, incluindo suas dificuldades sociais, conjugais, laborativas, de saúde; reflexões sobre seus valores e sentido da vida. Para tanto, foram utilizadas técnicas de Terapia CognitivoComportamental para auxílio no manejo de ansiedade e de Logoterapia para auxiliar os pacientes a lidarem melhor com suas limitações e dificuldades. Ao final das doze semanas, os pacientes cumpriram um total de 36 sessões de exercício, 36 sessões de reeducação respiratória, 12 sessões de psicoterapia e três sessões de educação. Neste momento, todos os pacientes foram reavaliados sob a forma de pós-teste. 
Os dados quantitativos foram descritos através da média e desvio padrão. Nas variáveis categóricas foi utilizado o percentual. Na comparação dos grupos de acordo com o tipo de variável analisada, foram utilizados os seguintes testes estatísticos: Teste t de Student não pareado, teste de qui-quadrado corrigido por Yates. O nível de significância adotado no estudo foi alfa=0,05. Os dados foram processados e analisados com o auxílio dos programas Statistical Package for the Social Sciences for Windows versão 6.0 (SPSS Inc. Chicago, IL, EUA) e Epi Info 2002.

\section{Resultados}

Entre os meses de julho de 2009 e junho de 2010, 17 pacientes integrantes de grupos de PRP foram avaliados nas formas de pré e pós-teste. Houve predominância do sexo masculino $(52,9 \%)$, idade média de 64,5 anos, $64,7 \%$ casados, $70,5 \%$ com nível de instrução de ensino fundamental. Em referência à situação ocupacional 82,2\% dos pacientes eram aposentados e, destes $21,4 \%$ por invalidez. A tabela 1 apresenta os dados demográficos.

\section{Tabela 1 - Dados demográficos}

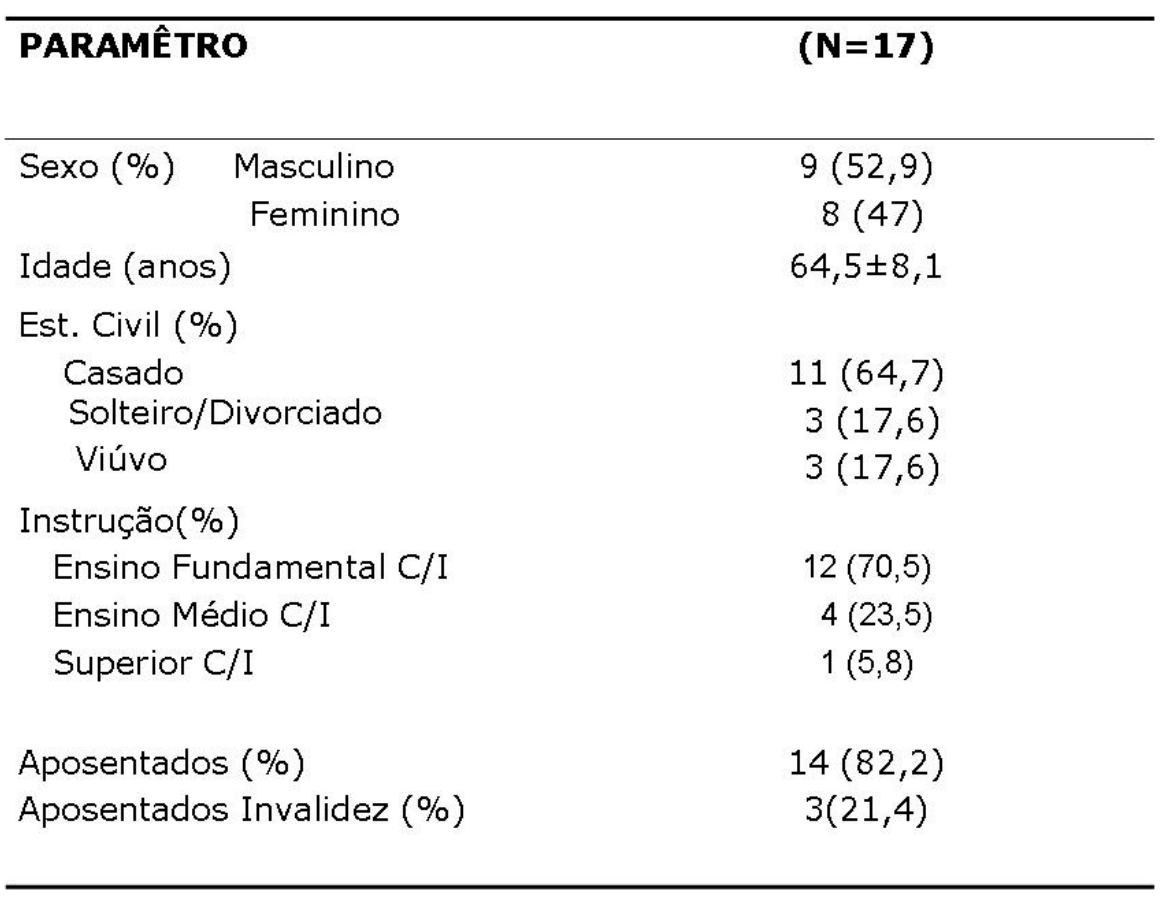

Os dados são apresentados em percentuais ou em média \pm desvio padrão. 
A avaliação inicial demonstrou que sintomas de ansiedade e depressão estiveram presentes em $29,4 \%$ da amostra, desesperança em $41,1 \%$.

Ao final do PRP a ansiedade não foi constatada na amostra como um critério significativo, apenas ocorreu num grau considerado mínimo; sintomas de depressão ocorreram em 5,8\% dos pacientes e desesperança em 17,6\%. Ver gráfico 1.

Gráfico 1- Comparação dos resultados percentuais de diagnóstico de sintomas de Ansiedade (BAI), Depressão (BDI) e Desesperança (BHS), no pré e pós-teste.

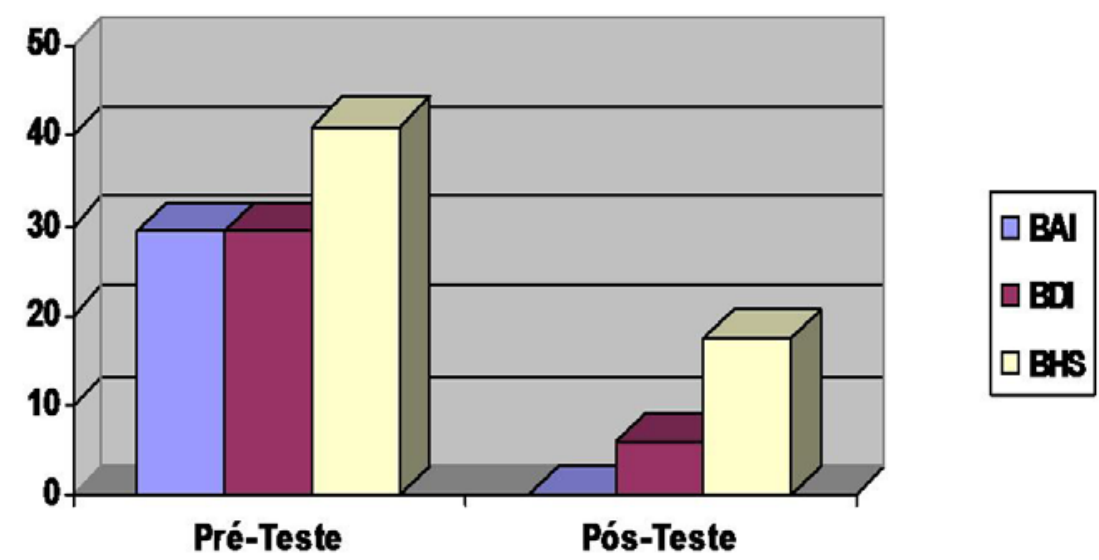

Na comparação entre as variáveis analisadas no pré e pós-teste, os pacientes demonstraram reduções estatisticamente significativas para os níveis de ansiedade, depressão e desesperança. Ver tabela 2.

Tabela 2 - Comparação dos resultados obtidos no pré e pós-teste para BAI, BDI e BHS 


\begin{tabular}{lccc}
\hline PARÂMetro & PRÉ -TESTE & Pós -Teste & $\boldsymbol{P}$ \\
\hline BAI & $7,2 \pm 6,4$ & $4 \pm 3,2$ & 0,002 \\
BDI & $8,5 \pm 2$ & $4 \pm 4$ & 0,001 \\
BHS & $5 \pm 5$ & $3 \pm 3$ & 0,03 \\
\hline
\end{tabular}

Os dados são apresentados em média tdesvio padrão. BAl: Inventário Beck de ansiedade; BDI: Inventário Beck de depressão; BHS: Escala de Desesperança de Beck

\section{Discussão}

Este estudo teve como objetivos avaliar o impacto do programa de reabilitação pulmonar (PRP) sobre os níveis de ansiedade, depressão e desesperança de pacientes com DPOC e demonstrar algumas contribuições que a Logoterapia pode oferecer para a compreensão desses pacientes.

Em relação às variáveis demográficas, esta investigação não se diferenciou dos resultados de outros estudos realizados. Há maior incidência de indivíduos do sexo masculino, com baixa escolaridade e estado civil casado, aposentados, na faixa etária de 60 anos de idade. Sendo, portanto, uma amostra composta por pacientes idosos GODOY; GODOY; BECKER; VACCARI; MICHELLI; TEIXEIRA, 2005).

Os níveis de ansiedade ocorreram num percentual de $29 \%$ dos pacientes. Índices aproximados aos encontrados nas investigações de Di Marco et al (2006) 28\% e Cleland, Lee \& Hall (2007) 32\%. Numa pesquisa com idosos os sintomas de ansiedade foram encontrados com freqüência. Sendo que, na maioria das vezes, a ansiedade veio associada a transtornos depressivos e a doenças físicas (BYRNE, 2002). Este aspecto foi corroborado no presente estudo, pois os pacientes apresentam DPOC e os níveis de ansiedade e depressão são mais elevados do que na população de idosos que não apresentam doenças clínicas (RABELO \& NERI, 2005).

O percentual de depressão, $29 \%$, encontra-se num nível similar ao do estudo de Janssen et al (2010) que encontrou sintomas de depressão em $27 \%$ dos casos de pacientes com DPOC. A depressão tem sido 
descrita como o transtorno que mais afeta o idoso e envolve uma série de comprometimentos na vida de uma pessoa. Indivíduos com depressão, de acordo com Beck (1997) apresentam a tríade cognitiva; tendência a avaliar negativamente a si mesmos, o mundo e o seu futuro.

Os resultados obtidos na comparação pré e pós-teste dessa investigação são corroborados por vários estudos realizados, demonstrando diminuição de ansiedade e depressão de pacientes com DPOC após a realização de PRP (GOLDBERG; HILLBERG; REI NECKER; GOLDESTEIN, 2004; GARUTI ; CILIONE ; DELL'ORSO ; GORINI ; LORENZI; TOTARO; CIRELLI ; CLINI, 2003; KAYAHAN; KARAPOLAT; ATYNTOPRAK; ATASEVER; OZTÜRK, 2006). No entanto, nenhuma pesquisa que evidenciasse a variável desesperança foi encontrada. Para a discussão serão trazidos alguns estudos com populações de idosos, especialmente os que apresentam algumas doenças crônicas.

$\mathrm{Na}$ amostra estudada, a desesperança foi observada em $41 \%$ dos pacientes. A desesperança tem sido relacionada, na velhice, ao sentimento de fracasso. Como o idoso não consegue realizar as tarefas com o mesmo sucesso com que realizava anteriormente, ele deixa de realizá-las. Neste ínterim, o sentimento de desesperança é inevitável. A velhice, especialmente quando associada a doenças, se caracteriza por sentimentos de fragilidade e impotência, pois evidencia-se uma perda de recursos físicos, mentais e sociais, que conduzem a sentimentos de desamparo (OLIVEIRA; ANGELI DOS SANTOS; CRUVINEL; NÉRI,2006).

Bryant et al. (2001) a partir de um estudo realizado com idosos, propuseram o que denominaram de modelo de envelhecimento saudável. Para a amostra investigada, saúde simbolizava fazer algo significativo, o que envolve quatro componentes: fazer algo que valha a pena fazer; apresentar um equilíbrio entre habilidades e desafios, possuir recursos sociais apropriados e características atitudinais positivas.

Quando se trata de populações clínicas e doenças incapacitantes, estes aspectos, raramente são preservados. Os programas de reabilitação devem buscar contribuir para que os pacientes consigam reestruturar suas vidas. A premissa básica dos programas de reabilitação era compreendida já em 1942, pelo Conselho da Associação Médica Americana de Reabilitação, como uma restauração do indivíduo, ao mais pleno potencial médico, mental, emocional, social e vocacional de que cada um é capaz (HODGKIN, 2009).

Superar sentimentos de impotência e desesperança são indispensáveis para que esses pacientes possam vislumbrar outras possibilidades. Na medida em que a pessoa puder reavaliar seus valores, abrir-se a outras perspectivas ela poderá ressignificar sua 
vida, apesar de, muitas vezes perceber a proximidade da morte (GODOY, 2003).

Beck (1997) refere que quando o paciente não vê razão para viver, não tem pelo que esperar, não consegue suportar a vida que tem, sente-se um fardo para a família, pode ver no ato suicida a única saída plausível. Esses aspectos apresentam alguma similaridade com a maneira como os pacientes com DPOC se sentem. Por isso, identificar e trabalhar os sentimentos de desesperança torna-se uma premissa tão importante para os programas de reabilitação em geral. Frankl (2008) refere que a busca de um significado para a vida é considerado um motor básico da existência humana. Elegendo o sentido como aspecto primordial da existência individual, ele criou a Logoterapia ou Psicoterapia do Sentido da Vida. De certa forma, o desespero e vazio existencial encontram-se em oposição à realização de sentido.

De acordo com Frankl (1987), o sentido poderá ser descoberto através da realização de valores denominados, valores de criação (trabalho), valores de vivência (amor) e valores de atitude (frente ao sofrimento, culpa e morte). Esta última categoria é considerada superior, pois possibilita ao homem transcender e superar situações de sofrimento intenso, consideradas inevitáveis, através da busca de um novo significado.

A logoterapia compreende que existem condicionamentos biopsicossociais. No caso deste estudo com pacientes com DPOC estes aspectos são bem identificados. No entanto, considera que na medida em que a pessoa puder refletir sobre suas circunstâncias ela poderá buscar um distanciamento de seu sintoma e, autotranscender-se, abrindo-se a novas possibilidades e encontrar novos valores. (FRANKL, 1987).

Vanistendael e Lecomte apud Silveira e Mahfoud (2008) afirmam que muitas pessoas, quando se defrontam com um fato traumático, lançam-se para fora de si, buscando a autotranscendência. Esse fato é visto pelos autores como uma fonte importante de resiliência.

Uma investigação realizada com pacientes com câncer em estágio terminal, concluiu que a depressão e desesperança apresentavam-se como preditores importantes do desejo de morte prematura na população estudada (BREITBART; ROSENFELD; PESSIN; KAIM; FUNESTI-ESCH; GALIETTA; NELSON; BRESCIA, 2000). Outra pesquisa encontrou correlação positiva entre depressão, ideação suicida e desesperança (CHOCHINOV; WILSON; ENNS; LANDER, 1998).

Baseado nos preceitos da Logoterapia, Breitbart et al. (2010) criaram uma abordagem grupal com duração de 8 sessões para tratar pacientes com câncer. Esta intervenção foi projetada para ajudar os pacientes com câncer avançado a manter ou melhorar um senso de 
significado, paz e propósito em suas vidas, e auxiliá-los no enfrentamento da morte.

A preocupação com a morte, o desejo da morte, o medo de sentir dor e de não conseguir suportar o sofrimento imposto por uma crise de dispnéia encontram-se no cerne da fala de vários pacientes com DPOC já estudados. (ANTUNES E MELLO FILHO, 2007).

Portanto, este estudo pode contemplar os objetivos inicialmente propostos. Foi possível observar, através da participação dos pacientes no PRP, uma diminuição expressiva dos sintomas de ansiedade, depressão e desesperança. Nas sessões de psicoterapia realizadas com auxílio dos preceitos da Logoterapia pode-se trabalhar temas como a ansiedade frente a morte, o sofrimento, a perda de valores e a redescoberta de novos valores. Situações que auxiliaram os pacientes a ressignificar suas vidas e a buscar novas perspectivas. Contudo nossos dados não permitem estimar se a melhora será duradoura após o término do PRP.

Recomenda-se que novos estudos possam aprimorar o método proposto nessa investigação, inserindo um número maior de pacientes para observar se os resultados encontrados são corroborados; verificar se os benefícios alcançados se mantêm ao longo do tempo e, pesquisar a variável desesperança com outros testes propostos na literatura, especialmente na área de logoterapia.

\section{Referências}

ANTUNES, AS, MELLO FILHO, J. Grupos com portadores de doença pulmonar obstrutiva crônica. In: Mello Filho, J. Grupo e corpo. Psicoterapia de grupo para pacientes somáticos. São Paulo: Casa do Psicólogo, 2007, p. 353-66.

BECK, A. Terapia cognitiva da depressão. Porto Alegre: ArtMed. 1997.

BREITBART $W$, ROSENFELD $B$, GIBSON C, PESSIN $H$, POPPITO $S$, NELSON C, TOMARKEN A, TIMM AK, BERG A, JACOBSON C, SORGER $B$, ABBEY J, OLDEN M. Meaning-centered group psychotherapy for patients with advanced cancer: a pilot randomized controlled trial. Psycho-Oncology, v. 19, n. 1, p. 21-8, Jan 2010. Disponível em: $<$ http://onlinelibrary.wiley.com/doi/10.1002/pon.1556/pdf >. Acesso em: 14 jan 2011.

BREITBART, W; ROSENFELD, B; PESSIN, H, KAIM, M; FUNESTIESCH,, J; GALIETTA, M; NELSON, CJ, BRESCIA, R. Depression, hopelessness, and desire for hastened death in terminally ill patients with cancer. J AMA, Chicago, v. 284, n. 22, p. 2907-2911, Dec. 2000. Disponível

em: <http://stage. web.fordham.edu/images/Undergraduate/psychology/al I\%20syllabus/rosenfeld/jama.paper.pdf>. Acesso em: 14 jan 2011. 
BRYANT, LL; KITTY K. CORBETT, KK; KUTNER J S. In their own words: a model of healthy aging. Social Science and Medicine, Oxford, $v$. 53, p. 297-41. 2001 Disponível em: <http://linkinghub. elsevier.com/retrieve/ pii/S0277953600003920>.

Acesso em: 24 jan 2011. Abstract.

BYRNE, G. What happens to anxiety disorders in later life? Revista Brasileira de Psiquiatria, Rio de Janeiro, v. 24, n. 1, p. 74-80. 2002. Disponível

em: <http://www.scielo.br/pdf/rbp/v24s1/8861.pdf>. Acesso em: 24 jan 2011

CELLI B.R. Predictors of mortality in COPD. Respiratory Medicine, cidade, London, v. 104, n. 6, p. 773-9, Jun 2010. Disponível em: <http://www.sciencedirect.com/science/article/ pii/S09546111100000 89> Acesso em: 12 jan 2011.

CHOCHINOV HM, WILSON KG, ENNS M, LANDER S. Depression, hopelessness, and suicidal ideation in terminally ill. Psychosomatics, Washington, v. 39, p. 366-370, 1998. Disponível em: $\quad$ <ttp://www.psy.psychiatryonline.org/cgi/reprint/39/4/366>. Acesso em: 12 jan 2011

CLELAND J A, LEE, AJ , HALL, S. Associations of depression and anxiety with gender, age, heath-related quality of life and symptoms in primary care COPD patients. Family Practice, Oxford, v. 24, n. 3, p. 217-23, 2007 Disponível em: <http://fampra.oxfordjournals.org/content/24/3/217.full.pdf+html>.

Acesso em: 12 jan 2011

CUNHA, J.A. Manual da versão em português das Escalas de Beck. São Paulo: Editora Casa do Psicólogo. 2001

DI MARCO, F.; VERGA, M.; REGGENTE, M.; MARIA CASANOVA, F.; SANTUS, P.; BLASI, F.; ALLEGRA, L.; CENTANNI, S. Anxiety and depression in COPD patients: the roles of gender and disease severity. Respiratory Medicine, London, v. 100, n. 10, p. 1767-74. $2006 . \quad$ Disponível em: <http://www.sciencedirect. com/science/article/pii/S09546111060006 06> Acesso em: 12 jan 2011

FRANKL, V.E. Ante EI vacío existencial. Hacia uma humanización de la psicoterapia. Barcelona: Herder, 1987.

FRANKL, V.E. La voluntad de sentido. Barcelona: Herder, 2008.

FRANKL, V.E. Psychotherapy and existentialism. Select papers on logotherepy. WSP: New York, 1985.

GARUTI G, CILIONE C, DELL'ORSO D, GORINI P, LORENZI MC, TOTARO L, CIRELLI G, CLINI E. I mpact of comprehensive pulmonary rehabilitation on anxiety and depression in hospitalized COPD patients. Monaldi Archives for Chest Disease, Brescia, v. 59, p. 56-61, 2003. Disponível em: <http://www.archest.fsm.it/pne/pdf/59/1/pne59-1_13garuti. pdf>. Acesso em: 12 jan 2011 
GODOY D.V.; GODOY R.F. A randomized, controlled trial of the effect of psychotherapy on anxiety and depression in chronic obstructive pulmonary disease. Archives of Physical Medicine and Rehabilitation, Philadelphia v. 84, p. 1154-57. 2003.

GODOY, D.V., GODOY, R.F., BECKER JR., B., VACCARI, P., MICHELLI, M., TEIXEIRA, P.Z. O efeito da assistência psicológica em um programa de reabilitação pulmonar para pacientes com doença pulmonar obstrutiva crônica. Jornal Brasileiro de Pneumologia, São Paulo, v. 31, n. 6, p. 499-505, 2005.

GODOY, R.F. Aspectos psicológicos do paciente em reabilitação. Medicina de Reabilitação, São Paulo, v. 22, n. 2, p. 21-22. 2003 GODOY, R.F.; TEIXEIRA, P.J.Z.; BECKER JR.; B., MICHELLI; M., GODOY, D.V. Repercussões tardias de um programa de reabilitação pulmonar sobre os índices de ansiedade, depressão, qualidade de vida e desempenho físico em portadores de DPOC. Jornal Brasileiro de Pneumologia, São Paulo, v. 35, n. 2, p. 129-36, 2009.

GOLDBERG R, HI LLBERG R, REINECKER L, GOLDESTEIN R. Evaluation of patients with severe pulmonary disease before and after pulmonary rehabilitation. Disability and Rehabilitation, London, $v$. 26, p. 641-8, 2004. Disponível em: <http://informahealthcare.com/doi/abs/10.1080/0963828041000166 3120>. Acesso em: 12 jan 2011. Abstract

HODGKIN, JE. Pulmonary rehabilitation: history and definition. In: Hodgkin, JE; Celli, BR; Connors, GL. Pulmonary rehabilitation: guidelines to success. 4th ed. Mosby/Elsevier.USA.2009, p. 1-7.

HUNG C.I.; LIU C.Y.; LIAO M.N.; CHANG Y.H.; YANG Y.Y.; YEH E.K. Self-destructive acts occurring during medical general hospitalization. General Hospital Psychiatry, New York, v. 22, n. 2, p. 115-21, Mar-Apr 2000. Disponível em: <http://www.ncbi.nlm.nih.gov/pubmed/10822098>. Acesso em: 20 jan 2011. Abstract

JANSSEN DJ; SPRUIT MA; LEUE C; GIJSEN C; HAMELEERS $H$; SCHOLS JM; WOUTERS EF. Symptoms of anxiety and depression in COPD patients entering pulmonary rehabilitation. Chronic Respiratory Disease, London, v. 7, n. 3, p. 147-57, Aug 2010. Disponível em: <http://crd.sagepub.com/content/7/3/147.short>. Acesso em 22 jan 2011.

KATZ P.P.; JULIAN L.J.; OMACHI T.A.; GREGORICH S.E.; EISNER M.D.; YELIN E.H.; BLANC P.D. The impact of disability on depression among individuals with COPD. Chest Journal, Chicago, v. 137, n. 4, p. 838-45, Apr 2010.2 Disponível em: <http://chestjournal.chestpubs.org/content/137/4/838.full. pdf+html $>$ Acesso em: 22 jan. 2011.

KAYAHAN, B.; KARAPOLAT, H.; ATYNTOPRAK, E.; ATASEVER, A.; OZTÜRK, O. Psychological outcomes of an outpatient pulmonary rehabilitation program in patients with chronic obstructive pulmonary 
disease. Respiratory Medicine, London, v. 100, n. 6, p. 1050-7. 2006.

<http://www.sciencedirect.com/science/article/pii/S09546111050040 99> Acesso em: 22 jan. 2011.

OLIVEIRA, KL; ANGELI DOS SANTOS, AA. CRUVINEL, M,NÉRI, AL. Relação entre ansiedade, depressão e desesperança entre grupos de idosos. Psicologia em Estudo, Maringá, v. 11, n. 2, p. 351-359, mai./ago. 2006. Disponível em: <http://www.scielo.br/pdf/pe/v11n2/v1ln2a13.pdf>. Acesso em 22 jan 2011.

OMACHI T.A.; KATZ P.P.; YELIN E.H.; IRIBARREN C.; KNIGHT S.J.; BLANC P.D.; EISNER M.D. The COPD Helplessness Index: a new tool to measure factors affecting patient self-management. Chest J ournal, Chicago, v. 137, n. 4, p. 823-30, Apr 2010. Disponível em: <http://chestjournal.chestpubs.org/content/137/4/823. full. pdf + h tml>. Acesso em: 22 jan 2011

RABE, K.F., HURD, S., ANZUETO, A., BARNES, P.J., BUIST, A.S., CALVERLEY, P. \& COLS. Global strategy for the diagnosis, management, and prevention of chronic obstructive pulmonary disease: GOLD executive summary. American Journal of Respiratory Critical Care Medicine, New York, v. 176, n. 6, p. 532-55. 2007.2 Disponível em: <http://171.66.122.149/cgi/content/full/176/6/532>. Acesso em 16 jul 2008.

RABELO, AF; NERI, AL. Recursos psicológicos e ajustamento pessoal frente à incapacidade funcional na velhice. Psicologia em Estudo, Maringá, v. 10, n. 3, p. 403-12, 2005. Disponível em: <http://www.scielo.br/pdf/pe/v10n3/v10n3a07.pdf>. Acesso em 22 jan 2011.

RIES, A.L., BAULDOFF, G.S., CARLIN, B.W., CASABURI, R., EMERY, C.F., MAHLER, D.A., \& COLS. Pulmonary rehabilitation: Joint ACCP/AACVPR evidence-based clinical practice guidelines. Chest J ournal, Chicago, v. 131, n. 5 Suppl, p. 4S-42S. 2007. Disponível em: <http://chestjournal.chestpubs.org/content/131/5_suppl/4S. Iong $>$. Acesso em: 20 jan 2011.

SILVEIRA, DR; MAHFOUD, M. Contribuições de Viktor Emil Frankl ao conceito de resiliência. Estudos de Psicologia; Campinas, v. 25, n. 4, p. 567-576, outubro - dezembro 2008. Disponível em: <http://www.scielo.br/pdf/estpsi/v25n4/a11v25n4.pdf>. Acesso em 22 jan 2011.

YORKE J; MOOSAVI S.H; SHULDHAM C; J ONES P.W. Quantification of dyspnoea using descriptors: development and initial testing of the Dyspnoea-12. Thorax- British Thoracic Society, Oxford, v. 65, n. 1 , p. 21-6, jan 2010. Disponível em: <http://thorax.bmj.com/content/65/1/21.full.pdf> Acesso em: 22 jan. 2011. 


\section{Endereço para correspondência Rossane Frizzo de Godoy}

Rua General Arcy da Rocha Nóbrega, 401 Sala 204, CEP 95040-000, Caxias do Sul - RS, Brasil.

Endereço eletrônico: rossanegodoy@terra.com.br

Recebido em: 16/09/2011

Reformulado em: 11/03/2013

Aceito para publicação em: 12/03/2013

Acompanhamento do processo editorial: Ana Maria Lopez Calvo de Feijoo

\section{Notas}

* Professora Adjunta - Centro de Ciências Humanas da Universidade de Caxias do Sul. Membro do Corpo Permanente do Núcleo de Pesquisa em Intervenções da Psicologia- Universidade de Caxias do Sul. Brasil. Psicóloga; Doutora em Ciências Pneumológicas (UFRGS); Mestre em Ciências do Movimento Humano (UFRGS), Especialista em Psicoterapia Humanístico-Existenciais: Logoterapia (PUC-RS). 